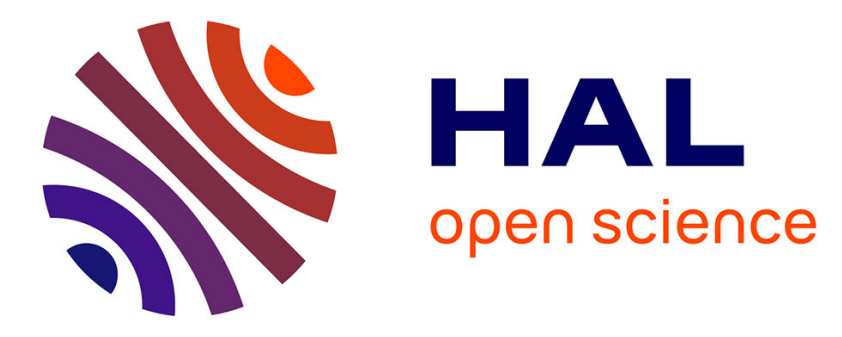

\title{
Improvements of Adaptive Filtering by Optimal Projection to filter different artifact types on long duration EEG recordings
}

Samuel Boudet, Laurent Peyrodie, Gérard Forzy, Antonio Pinti, Hechmi Toumi, Philippe Gallois

\section{To cite this version:}

Samuel Boudet, Laurent Peyrodie, Gérard Forzy, Antonio Pinti, Hechmi Toumi, et al.. Improvements of Adaptive Filtering by Optimal Projection to filter different artifact types on long duration EEG recordings. Computer Methods and Programs in Biomedicine, 2012, 108 (1), pp.234-49. 10.1016/j.cmpb.2012.04.005 . hal-00804652

\section{HAL Id: hal-00804652 https://hal.science/hal-00804652}

Submitted on 26 Mar 2013

HAL is a multi-disciplinary open access archive for the deposit and dissemination of scientific research documents, whether they are published or not. The documents may come from teaching and research institutions in France or abroad, or from public or private research centers.
L'archive ouverte pluridisciplinaire HAL, est destinée au dépôt et à la diffusion de documents scientifiques de niveau recherche, publiés ou non, émanant des établissements d'enseignement et de recherche français ou étrangers, des laboratoires publics ou privés. 


\title{
Improvements of Adaptive Filtering by Optimal Projection to filter different artifact types on long duration EEG recordings
}

\author{
S. Boudet ${ }^{\mathrm{a}, \mathrm{b}, \mathrm{c}, \mathrm{d}}$, L. Peyrodie ${ }^{\mathrm{a}, \mathrm{e}, \mathrm{f}, \mathrm{d}}$, G. Forzy ${ }^{\mathrm{a}, \mathrm{b}, \mathrm{c}, \mathrm{d}}$, A. Pinti ${ }^{\mathrm{a}, \mathrm{g}}$, H. Toumi ${ }^{\mathrm{h}, \mathrm{g}}$, \\ P. Gallois ${ }^{\mathrm{a}, \mathrm{b}, \mathrm{c}, \mathrm{d}}$ \\ ${ }^{a}$ Univ Nord de France, F-59000 Lille, France \\ ${ }^{b}$ UCLille, F-59000 Lille, France \\ ${ }^{c}$ Groupe Hospitalier de l'Institut Catholique Lillois/ Faculté Libre de Médecine, F-59000 \\ Lille, France \\ ${ }^{d}$ Unité de Traitement de Signaux Biomédicaux, 59000 Lille, France \\ ${ }^{e}$ Hautes Etudes d'Ingénieur, 13 rue de Toul, 59000 Lille, France \\ ${ }^{f}$ Laboratoire d'Automatique, Génie Informatique et Signal (LAGIS FRE CNRS 3303), \\ 59650 Villeneuve d'ascq, France \\ ${ }^{g}$ LAMIH (FRE CNRS 3304), Université de Valenciennes, France \\ ${ }^{h}$ Institut de Prévention et de Recherche sur l'Ostéoporose (IPROS INSERM U658), \\ Université d'Orléans, France
}

\begin{abstract}
Adaptive Filtering by Optimal Projection (AFOP) is an automatic method for reducing ocular and muscular artifacts on electro-encephalographic (EEG) recordings. This paper presents two additions to this method: an improvement of the stability of ocular artifact filtering and an adaptation of the method for filtering electrode artifacts. With these improvements, it is possible to reduce almost all the current types of artifacts, while preserving brain signals, particularly those characterising epilepsy. This generalised method consists in dividing the signal into several time-frequency windows, and in
\end{abstract}

Email address: samuel.boudet@gmail.com (S. Boudet)

$U R L:$ http://www. samuelboudet.com (S. Boudet) 
applying different spatial filters to each. Two steps are required to define one of these spatial filters: the first step consists in defining artifact spatial projection using the Common Spatial Pattern (CSP) method and the second consists in defining EEG spatial projection via regression. For this second step, a progressive orthogonalisation process is proposed to improve stability. This method has been tested on long-duration EEG recordings of epileptic patients. A neurologist quantified the ratio of removed artifacts and the ratio of preserved EEG. Among the 330 artifacted pages used for evaluation, readability was judged better for $78 \%$ of pages, equal for $20 \%$ of pages, and worse for $2 \%$. Artifact amplitudes was reduced by $80 \%$ on average. At the same time, brain sources were preserved in amplitude from $70 \%$ to $95 \%$ depending on the type of waves (alpha, theta, delta, spikes, ... ). A blind comparison with manual Independent Component Analysis (ICA) was also realised. The results show that this method is competitive and usefull for routine clinical practice.

Keywords:

EEG, Automatic filtering, Muscle artifacts, Ocular artifacts, Electrode artifacts, Epilepsy, CSP

\section{Introduction}

The electroencephalogram (EEG) is a fundamental examination in neurology. It is especially important in epileptology since it enables screening for paroxysmal activities. Particularly, analysis of signals recorded during seizures, provides information concerning the localistion and characterisation of the epileptic disorder usefull for planing medical or surgical treatment. 

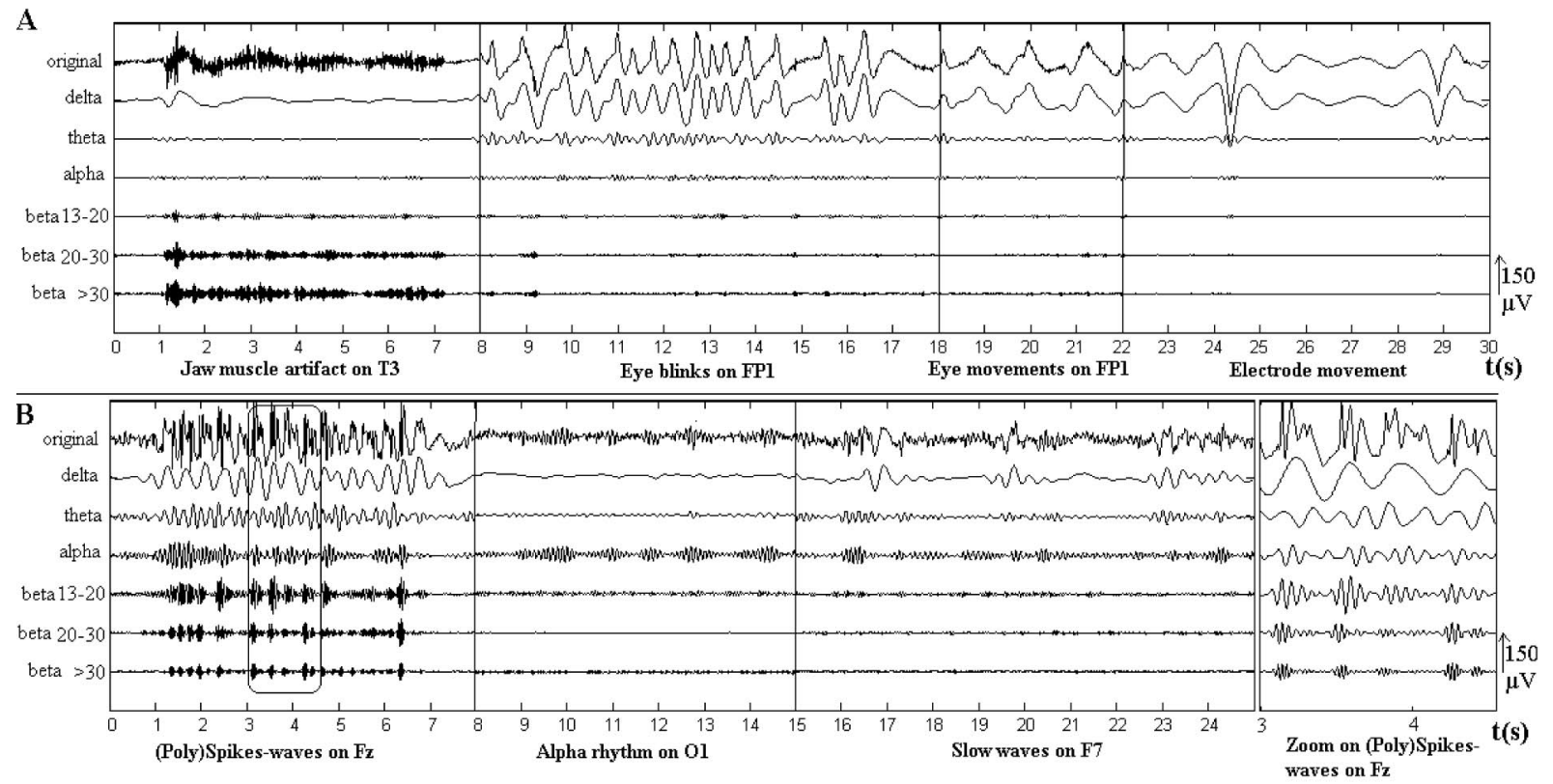

Figure 1: Frequency band decomposition of (A) artifacts and (B) brain signals

Unfortunately, parasitic signals called artifacts can easily be contaminate EEG activity compromising interpretation. These artifacts can be broken down into three categories:

- Muscular artifacts correspond to the electro-myographic potentials generated by muscles, mainly in the jaw and forehead. Recorded in the beta band, these artifacts can be diffuse, but are more generally found in the frontal and temporal areas (Fig. 1-A).

- Ocular artifacts resulting from the mechanical movement of the eyes or the eyelids contaminate mainly the delta band (Fig. 1-A), again in the frontal and temporal areas. They may be symmetrical or antisymmetrical between the two hemispheres depending on their origin (eye blink, horizontal/vertical eye movement) 
- Electrode artifacts are also slow waves found mainly in the delta band. These artifacts are created by electrode or wire movements, skin galvanic resistant variation or heart beats. Their amplitude can be very high and they are often recorded by only one lead. As a rule, muscle artifacts come with electrode artifacts triggered by the facial movement resulting from the muscle contraction (Fig. 1-A time: 1s to 2s).

There is also the sector artifact, generally removed by a notch filter, and the electro-cardiographic artifact, which though uncommon can be filtered using the method described by Wang et al.[1] [2].

The purpose of this paper is to present a filter designed to:

- reduce the most common and problematic types of artifacts (ocular, muscular and electrode-relateds);

- preserve the brain rhythms and particularly those characterising epilepsy (spike, spike-waves and slow waves) (Fig. 1-B);

- avoid any requirement for manual intervention.

This paper starts by describing the background of artifact filtering. Next, the method and theoretical aspects of the filtering are described. Finally, the results are presented and blindly compared to those obtained with Independent Component Analysis (ICA).

\section{Background}

\subsection{Earlier work on artifact filtering}

As shown in Figure 1, the frequencies of pathological graphic-elements overlap those of artifacts, thus making it impossible to use frequency filters 
(e.g. lowpass, highpass, wavelets, ... ) to separate them. Consequently, research in this field has been oriented towards spatial filters.

The first methods were based on Principal Component Analysis [3] and on regression (for review see [4]). Then, much progress was made with the development of ICA that seems to give better results [5]. In order to filter artifacts, each ICA component has to be identified as an artifact source or as a brain source. One of the major problems of ICA is that the identification is often performed manually by a specialist. This step can tedious and timeconsuming. Much research has been devoted to automating this procedure [6][7], or to adapt the ICA base to better fit artifact and brain sources [8].

Most artifact filtering methods aim to filter only one type of artifact (ocular, muscular, cardiac) and only a very few of them have been tested in presence of pathological activity (slow waves, spikes, etc.). Combing filters often results in a deletion of a major part of the cerebral rhythm especially pathological signals. P. Levans et al. [9] presented a rigorous study on this subject examining all types of artifacts. They describe a method that identifies each component with a Bayesian classifier. Applying this method to seizure recordings demonstrates that it often improves readability, but at the cost of great reduction in the brain signal without complete removal of all artifacts when they are numerous.

\subsection{Recent contributions}

In the same context, we developed the Adaptive Filtering by Optimal Projection (AFOP) method [10] and observed its good performance in a preliminary study of short-duration recordings in epileptic patients. Nevertheless, since the subjects were asked not to move, these short duration 
recordings were less affected by artifacts than long-duration recordings. In addition, no seizures were studied.

Consequently, the AFOP in its present form has two limitations:

- Electrode-related artifacts cannot be filtered and in the presence of those artifacts, the the filter effect could be random;

- stability could be a problem when filtering ocular artifacts.

The following paper reports two improvements that address these limitations: the first one called the Dual AFOP method (DAFOP) is designed to filter electrode artifacts; the second one called progressive orthogonalisation, is designed to stabilise the method. In addition, compared with our earlier report [10], we have provided a more general presentation of the method, making the introduction of these two improvements more logical.

\section{Filtering method}

\subsection{Summarizing the overal process}

The overall process is a combination of spatial filtering and frequency filtering.

Each of the spatial filters is a combination of at least one of the following three processes:

- A process to remove muscular artifacts based on the AFOP method[10].

- A process to remove ocular artifacts also based on the AFOP method[10] as well as on the progressive orthogonalisation process, introduced in this paper for stabilisation (sec. 3.5). 
- A process to remove electrode artifacts which is based on DAFOP dual frequency method, introduced in this paper.

The entire recording is the rebuilt by summing the spatially filtered windows.

The AFOP method[10] consists in determining the spatial distribution of artifacts using to a learning period (sec. 3.4.1).

The DAFOP method consists in optimising the frequency pattern of each component to better fit brain signals (sec. 3.4.1) and artifacts (sec. 3.4.2).

The overall method is illustrated in Figure 2. The overall method is based on spatial filtering which must first be defined.

\subsection{Spatial filtering}

Spatial filterings consists in creating some linear combinations of the signals from the various channels in order to reduce the amount of artifacts.

Considering $\mathbf{V}$ (dimension $n \times t$ ), the signal matrix where lines correspond to $n$ channels and columns to $t$ time samples, the linear combinations can be represented by a filtering matrix $\mathbf{F}$ (dimension $n \times n$ ) which is an oblique projector (i.e. $\mathbf{F F}=\mathbf{F}$ ) [10]. The filtered signal can be obtained by:

$$
\mathbf{V}^{\prime}=\mathbf{F V}
$$

with $\mathbf{V}^{\prime}$ representing the filtered signals.

The projection matrices are diagonalisable and the eigenvalues are all 1 or 0 . The diagonalisation can be written $\mathbf{F}=\mathbf{M D M}^{-1}=\mathbf{W}^{-1} \mathbf{D W}$ with $\mathbf{D}$ $(n \times n)$ a diagonal matrix composed by 1 on the first $n_{1}$ diagonal elements and by 0 on the last $n_{0} . \mathbf{M}(n \times n)$ is the matrix of the right eigenvectors 
and $\mathbf{W}=\mathbf{M}^{-1}(n \times n)$ is the matrix of left eigenvectors. By analogy with blind source separation theory[11][5], $\mathbf{W}$ is called a separation matrix, $\mathbf{M}$ is called a mixing matrix and the matrix $\mathbf{S}=\mathbf{M V}(n \times t)$ is called a source matrix.

A filtering matrix is defined in an unique way by two eigenspaces $E_{1}$ and $E_{0}$ of respective dimension $n_{1}$ and $n_{0}\left(n_{1}+n_{0}=n\right)$. The subspace $E_{1}$ (corresponding to eigenvalue 1) is the image of $\mathbf{F}\left(E_{1}=\left\{\mathbf{x} \in \Re^{n} \backslash \mathbf{F x}=\mathbf{x}\right\}\right.$ ) and the subspace $E_{0}$ (corresponding to eigenvalue 0$)$ is the nullspace $\left(E_{0}=\right.$ $\left.\left\{\mathbf{x} \in \Re^{n} \backslash \mathbf{F} \mathbf{x}=\mathbf{0}\right\}\right)$.

The first $n_{1}$ column vectors of $\mathbf{M}$ are a base of the subspace $E_{1}$ and the last $n_{0}$ are a base of $E_{0}$. In the same way, the first $n_{1}$ line vectors of $\mathbf{W}$ are a base of the subspace $E_{0}^{\perp}$ (orthogonal subspace of $E_{0}$ ) and the last $n_{0}$ line vectors of $\mathbf{W}$ are a base of the subspace $E_{1}^{\perp}$.

$E_{1}$ is then called the mixing subspace of sources of interest (in the case of EEG, it represents the distribution of brain sources on the various electrodes), $E_{0}$ is called the mixing subspace of artifacts, $E_{1}^{\perp}$ is called the separation subspace of artifacts and $E_{0}^{\perp}$ is called the separation subspace of sources of interest. The filtering matrix can thus be constructed using any base $\mathbf{M}_{0}$ of $E_{0}$ or $\mathbf{W}_{1}^{T}$ of $E_{0}^{\perp}$ and any base $\mathbf{M}_{1}$ of $E_{1}$ or $\mathbf{W}_{0}^{T}$ of $E_{1}^{\perp}$ and applying one of the following formulas: 


$$
\begin{aligned}
\mathbf{F} & =\mathbf{M}_{1}\left(\mathbf{W}_{1} \mathbf{M}_{1}\right)^{-1} \mathbf{W}_{1} \\
& =\mathbf{I}_{n}-\mathbf{M}_{0}\left(\mathbf{W}_{0} \mathbf{M}_{0}\right)^{-1} \mathbf{W}_{0} \\
& =\left(\frac{\mathbf{W}_{1}}{\mathbf{W}_{0}}\right)^{-1} \mathbf{D}\left(\frac{\mathbf{W}_{1}}{\mathbf{W}_{0}}\right) \\
& =\left(\mathbf{M}_{1} \mid \mathbf{M}_{0}\right) \mathbf{D}\left(\mathbf{M}_{1} \mid \mathbf{M}_{0}\right)^{-1}
\end{aligned}
$$

Only this first formula was used in [10] since the AFOP process automatically defines $\mathbf{W}_{1}$ and $\mathbf{M}_{1}$. In order to simplify the introduction of the stabilisation process (sec. 3.5.5), a more general approach can be achieved by constructing the filtering matrix using subspaces instead of matrices. Consequently, any one of those formulas can be used depending of the subspaces involved.

\subsection{Time-frequency decomposition}

One of the limitations of spatial filtering is that sources cannot be completely separated if the number of sources is greater than the number of channels. It is obvious in practice that there are many more sources than channels even though most of the signal power may be contained in only a few components. One way to increase the number of components is to use the specific properties of sources which exhibit both frequential and temporal parsimony. Indeed, artifacts and brain rhythms are never activated over the entire recording. In addition, they are confined to a small part of the Fourier spectrum (Fig. 1).

In order to take this parsimony effect into account, the EEG's multiple channels can be broken down into several time-frequency windows $\mathbf{V}^{\phi}$. 
A time-frequency window corresponds to the signal of a time window filtered by a band pass filter of a frequency window. It should also be noted that the entire process can work within the framework of Fourier analysis, all steps of the AFOP/DAFOP method giving exactly the same results because of the linear properties and the Parceval relation. In this case, a time frequency windows would correspond to the signal of a frequency window filtered by a band pass filter of a time window.

The purose of the time-frequency decomposition process is to apply a different spatial filter $\mathbf{F}^{\phi}$ on each of these time-frequency windows. The entire filtered EEG will then correspond to the sum of all these windows filtered with their specific filtering matrix. This process is equivalent to the frequency band decomposition described in [10]).

The frequency windows used for this decomposition are set to the traditional bands used for electroencephalography (except for beta which is divided) $\left(\Delta: 0-4 H z, \theta: 4-8 H z, \alpha: 8-13 H z, \beta_{1}: 13 H z-20 H z, \beta_{2}\right.$ : $20 \mathrm{~Hz}-45 \mathrm{~Hz}$ ). These windows are chosen to best isolate either brain rhythms or artifacts.

It is recommended to achieve this decomposition by applying a Perfect Reconstruction Filter Bank [12]. The important point is that the entire EEG has to correspond exactly to the sum of all these time-frequency windows. A zero-phase filter (e.g. forward-backward filter, Fourier filter or centred Finite Impulse Response filter) should be applied to perfectly comply with this requirement. The method used in this paper corresponds to a cascade of 6-order low-pass butterworth filters with a forward-backward process.

The aim is now to construct each of those spatial filters $\mathbf{F}^{\phi}$ which will be 
applied independently on the time-frequency windows $\mathbf{V}^{\phi}$. A combination of AFOP and DAFOP methods are used to generate thes filtering matrices, depending of the type of artifacts to be filtered in the window.

\subsection{The AFOP/DAFOP method}

Both methods AFOP and DAFOP, consist in a two-step construction of the filtering matrix $\mathbf{F}^{\phi}$ for a window $\mathbf{V}^{\phi}$ : the first step defines $E_{0}^{\phi}$ and the second $E_{1}^{\phi}$.

\subsubsection{Common Spatial Pattern: learning $E_{0}$}

The first step is to define two training windows: $\mathbf{V}_{i m p}\left(n \times T_{i m p}\right)$ and $\mathbf{V}_{\text {art }}\left(n \times T_{\text {art }}\right) . \quad \mathbf{V}_{\text {imp }}$ is defined to contain mostly signals of interest and $\mathbf{V}_{\text {art }}$ is defined to contain mostly artifacts. Those two windows are used to determine the brain source separation, which is equivalent to the artifact distribution. This artifact distribution will then be considered as constant for the current window $\mathbf{V}^{\phi}\left(n \times T^{\phi}\right)$.

The subspace is determined by the identifying components whose variance increases the least between $\mathbf{V}_{i m p}$ and $\mathbf{V}_{\text {art }}$. The two methods, AFOP and DAFOP, differ by the way they chose these windows depending on the type of artifact to filter:

a. The AFOP method[10]: If the artifact distribution (e.g. eyes or muscles) is considered constant over time, $\mathbf{V}_{\text {imp }}, \mathbf{V}_{\text {art }}$ and $\mathbf{V}$ can be chosen to correspond to the different time windows. The eyes and the head muscles have a constant location in the head, imposing a corresponding artifact distributions. This artifact distribustion can be learned by asking the subject to perform a protocol [10] composed of a set of standard movements (eye blinks, 
eye movements, jaw contractions, forehead contractions, smiles). According to our trials, this set of movements seemed enough to filter almost all types of artifacts except electrode artifacts.

The brain and artifact sources could be considered static since the brain and the muscles are always in the same location. Some sources might however not be activated during the rest period of the learning step. For example, sources of paroxysms may not appear in this period. Consequently, only the artifact distribution space will be considered as constant.

b. The DAFOP method: If the artifacts have specific frequential spectrum difference compared to important sources and if the artifact spatial distribution is the same for all frequencies, (e.g. electrode artifacts), $\mathbf{V}_{\text {imp }}, \mathbf{V}_{\text {art }}$ and $\mathbf{V}^{\phi}$ can be chosen to correspond to different frequency windows.

The AFOP method can be used for the filtering of artifacts with a constant distribution. This is the case for ocular and muscular artifacts but not for electrode artifacts. Indeed, for example, a wire movement can appear on any electrode at any time. However, these artifacts are characterised by their very low frequencies even compared to delta rhythms. Particularly, the spectral power is near null for frequencies exceeding $4 \mathrm{~Hz}$ (Fig. 1-A).

To filter these artifacts, a Common Spatial Pattern (CSP) process can be carried out between two frequency bands [13], [14] empirically defined. On the current sliding time window, the signals of the band $0.5-2 \mathrm{~Hz}$ are compared to the signals of the band $4-8 \mathrm{~Hz}$. The components having the greatest variance ratio between those two frequency bands are considered as electrode artifact sources. 
c. Resolution. Once the two windows are defined, the aim is to find components whose variance increases the least between $\mathbf{V}_{i m p}$ and $\mathbf{V}_{\text {art }}$. This problem can be solved by CSP processing.

This consists in computing the two covariance matrices $\mathbf{C}_{i m p}=\frac{\mathbf{V}_{i m p} \mathbf{V}_{i m p}{ }^{T}}{T_{i m p}}$ and $\mathbf{C}_{\text {art }}=\frac{\mathbf{V}_{\text {art }} \mathbf{V}_{\text {art }}{ }^{T}}{T_{\text {art }}}$. The subspace $E_{0}^{\perp}$ corresponds to the components w with the smallest variance ratio $r(\mathbf{w})$ between $\mathbf{V}_{\text {art }}$ and $\mathbf{V}_{\text {imp }}(r(\mathbf{w})=$ $\left.\frac{\left\|\mathbf{w} \mathbf{V}_{\text {art }}\right\|^{2}}{\left\|\mathbf{w} \mathbf{V}_{\text {imp }}\right\|^{2}}\right) . \quad E_{0}^{\perp}$ is then the subspace generated by the first $n_{1}$ eigenvectors $\left(\left[\mathbf{w}_{1}, \mathbf{w}_{2}, \ldots, \mathbf{w}_{n_{1}}\right]=\mathbf{W}_{1}^{T}\right)$ corresponding to the smallest eigenvalues of $\mathbf{C}_{i m p}{ }^{-1} \mathbf{C}_{\text {art }}$. The diagonalisation of $\mathbf{C}_{i m p}{ }^{-1} \mathbf{C}_{\text {art }}$ can be written:

$$
\mathbf{C}_{i m p}{ }^{-1} \mathbf{C}_{a r t}=\mathbf{W}^{T} \mathbf{D}_{C S P} \mathbf{M}^{T}
$$

with $\mathbf{D}_{C S P}$ being the diagonal matrix of eigen values $\lambda_{i}(i=1 \ldots n), \mathbf{W}=$ $\left(\frac{\mathbf{W}_{1}}{\mathbf{W}_{0}}\right)$ being the transpose matrix of eigen vectors and $\mathbf{M}=\mathbf{W}^{-1}=$ $\left(\mathbf{M}_{1} \mid \mathbf{M}_{0}\right)$ being the inverse matrix. It can be shown that the eigenvalues $\lambda_{i}$ correspond to the component variance ratios $r\left(\mathbf{w}_{i}\right)$ between the two periods.

There is the property that the subspace $E_{0}^{\perp}$ of bases $\mathbf{W}_{1}^{T}$ and the subspace $E_{1}^{\perp}$ of bases $\mathbf{W}_{0}^{T}$ are orthogonal for both scalar products $<\mathbf{C}_{i m p} ., .>$ and $<\mathbf{C}_{a r t}, .,>$. Similarly, the subspace $E_{0}$ of bases $\mathbf{M}_{0}$ and the subspace $E_{1}$ of bases $\mathbf{M}_{1}$ are orthogonal for both scalar products $<\mathbf{C}_{i m p}{ }^{-1}$.,. $>$ and $<\mathrm{C}_{\text {art }}{ }^{-1}, .,>$.

There are two possibilities to determine the dimensions $n_{1}$ and $n_{0}=$ $n-n_{1}$. First, if the number of sources to isolate artifact $n_{1}$ is unknown (e.g. for muscular artifacts), it can be automatically determined by selecting only 
eigenvectors $\mathbf{w}_{i}$ whose eigenvalues $\lambda_{i}$ are below a threshold $t_{C S P}$. Second, if these sources can be specifically localised (e.g. for ocular artifacts), the dimension $n_{0}=n-n_{1}$ can be set to a constant number of artifact sources.

\subsubsection{Regression: determining $E_{1}$}

Once the subspace $E_{0}^{\perp}$ is defined, the subspace $E_{1}^{\phi}$ is determined by finding the best distribution of sources of interest by a least squares method on the sliding window $\phi[10]$ of the signal matrix $\mathbf{V}^{\phi}$. Another way to consider this step is to conserve most signals exhibiting a distribution not exactly identified as artifact. This best distribution is defined by $\mathbf{M}_{1}^{\phi}=$ $\operatorname{argmin}_{\mathbf{M}_{1}} \sum_{i}\left\|\mathbf{V}_{i}^{\phi}-\mathbf{M}_{1} \mathbf{W}_{1} \mathbf{V}_{i}^{\phi}\right\|^{2}\left(\mathbf{V}_{i}^{\phi}\right.$ means the $i^{\text {th }}$ column of $\left.\mathbf{V}^{\phi}\right)$ and can be computed with:

$$
\mathbf{M}_{1}^{\phi}=\mathbf{C}^{\phi} \mathbf{W}_{1}^{T}\left(\mathbf{W}_{1} \mathbf{C}^{\phi} \mathbf{W}_{1}^{T}\right)^{-1}
$$

Where $\mathbf{C}^{\phi}=\frac{\mathbf{V}^{\phi} \mathbf{V}^{\phi^{T}}}{T^{\phi}}$ is the covariance matrix. Consequently, $\mathbf{F}^{\phi}=$ $\mathbf{M}_{1}^{\phi} \mathbf{W}_{1}$. It can be noticed that if the period $\mathbf{V}^{\phi}$ corresponds to one of the period $\mathbf{V}_{i m p}$ or $\mathbf{V}_{\text {art }}$, then $\mathbf{M}_{1}^{\phi}=\mathbf{M}_{1}$ of eq. 3 in both cases. This can be explained by the fact that this step of regression consists in defining $E_{1}^{\phi}$ as the $\mathbf{C}^{\phi^{-1}}$-orthogonal subspace of $E_{0}$.

$$
E_{1}^{\phi}=E_{0}^{\perp} \mathbf{C}^{\phi^{-1}}
$$

\subsection{Stabilisation by progressive orthogonalisation}

\subsubsection{Stability problem}

There can be a stability problem if the artifact distribution is slightly different from that occurring during the learning period. This problem is 
common when the sources of interest have a small magnitude compared to the artifact sources. The regression step focuses on a small artifact residue in the estimation of sources of interest. The components wearing this artifact residue are highly amplified in order to rebuild them, resulting in an over-amplification of the signal of interest present in these components. For the EEG, this problem often occurs with ocular artifacts on the ? band. The covariance matrix can be easily misevaluated due to the low frequency and low amplitude of brain signals, producing incorrect signals on frontal electrodes (e.g. Fig. 4-C).

\subsubsection{Detection of instability}

This instability can be detected by a small minimum angle between the two subspaces $E_{1}^{\phi}$ and $E_{0}$. To get an idea of the meaning of angles between two subspaces $A$ and $B$ with dimensions $n_{A}$ and $n_{B}$, it must be understood that there are $\min \left(n_{A}, n_{B}\right)$ angles between these subspaces. If all these angles are null, this is equivalent to one of these subspaces being included in the other, and if at least one angle is null it is equivalent to $A \cap B \neq\{0\}$. The following equalities can be described:

$$
\begin{aligned}
\theta & =\operatorname{MinAngle}(A, B) \\
& =\frac{\pi}{2}-\operatorname{MaxAngle}\left(A, B^{\perp}\right) \\
& =\arcsin \frac{1}{\left\|\mathbf{F}_{A, B}\right\|_{2}}
\end{aligned}
$$

with $\mathbf{F}_{A, B}$ the oblique projector of null subspace $B$ and image $A$. The $\|\mathbf{F}\|_{2}$ corresponds to the usual norm for matrix defined as $\|\mathbf{F}\|_{2}=\max _{\mathbf{x}} \frac{\|\mathbf{F x}\|_{2}}{\|\mathbf{x}\|_{2}}$. 
A small angle between $E_{1}^{\phi}$ and $E_{0}$ corresponds to a high norm of the filtering matrix $\mathbf{F}^{\phi}$. This means that a signal with a small amplitude can be highly amplified and some instability is probable.

\subsubsection{Principal component projection}

To reduce this problem, the degrees of freedom can be reduced during the regression step. The process consists in applying Principal Component Analysis (PCA) to the current window $\phi$. The subspace $E_{0}$ is slightly displaced by an orthogonal projection on the principal component subspace $\left(E_{0}^{\phi, k}=\mathbf{P}_{P C A_{k}^{\phi}}^{\perp}\left(E_{0}\right)\right.$ with $\mathbf{P}_{P C A_{k}^{\phi}}^{\perp}$ being the application of orthogonal projection on the $k$-first principal components). The regression step then takes place strictly within this principal component subspace (called $E_{P C A_{k}}$ ).

For the last components of the PCA, it is not possible to distinguish what is artifact and what is cerebral. The choice is made to keep those components by adding them to $E_{1}^{\phi}$. This process is then exactly equivalent to the process: project $E_{0}$ on the principal component subspace and perform the regression step on the original base:

$$
E_{1}^{\phi, k}=\left(\mathbf{P}_{P C A_{k}^{\phi}}^{\perp}\left(E_{0}\right)\right)^{\perp_{\mathbf{C}^{-1}}}
$$

\subsubsection{The number of principal components $k$}

The number of principal components $(k)$ declines from $n$ until the mini-

mum angle between the two subspaces $\left(E_{0}^{\phi, k}\right.$ and $\left.E_{1}^{\phi, k}\right)$ would be greater than a threshold (e.g. $20^{\circ}$ for EEG).

At the beginning, (i.e. $k=n$ ) this method is equivalent to the standard $\mathrm{AFOP}[10]$. If there is no stability problem, there is a high probability that 
the minimum angle would be greater than the threshold and the orthogonalisation process would not start[10].

In the worst case, $k$ reaches $n_{0}$. Then, $E_{0}^{\phi, n_{0}}$ corresponds to the subspace generated by the $n_{0}$ first principal components $\left(E_{P C A_{n_{0}}}\right)$ and $E_{1}^{\phi, n_{0}}$ corresponds to the subspace generated by the $n_{1}$ last principal components. Consequently, $E_{1}^{\phi, n_{0}}$ would be the orthogonal subspace of $E_{0}^{\phi, n_{0}}$, the minimum angle would be equal to $\pi / 2$ and the algorithm stops. The filtering matrix is then the orthogonal projector that removes the first $n_{0}$ PCA components and keeps the last $n_{1}$. This is why this process is called progressive orthogonalisation.

\subsubsection{Precise algorithm}

Here is the algorithm of progressive orthogonalisation:

Input: $\mathbf{V}^{\phi}$ the signal matrix of the current sliding window $t, E_{0}$ the artifact mixing subspace learned with section 3.4.1

Output: The $\mathbf{F}^{\phi}$ projector matrix for the window $t$

1. PCA: Compute the covariance matrix and its diagonalisation $\mathbf{C}^{\phi}=$ $\mathbf{Q}^{\phi} \mathbf{D}_{\mathbf{C}^{\phi}} \mathbf{Q}^{\phi^{-1}}$ (with $\mathbf{D}_{\mathbf{C}^{\phi}}$ diagonal matrix of eigen values sorted in decreasing order)

2. Set $k=n$

3. Define $E_{P C A_{k}}$ as the subspace generated by the $k$ first column vectors of $\mathbf{Q}^{\phi}$

4. Define $E_{0}^{\phi, k}$ as the orthogonal projection of $E_{0}$ on $E_{P C A_{k}}\left(E_{0}^{\phi, k}=\right.$ $\left.\mathbf{P}_{P C A_{k}^{\phi}}^{\perp}\left(E_{0}\right)\right)$

5. Define $E_{1}^{\phi, k}$ as the $C^{\phi^{-1}}$-orthogonal subspace of $E_{0}^{\phi, k}$ (Regression) 
6. If the minimum angle between $E_{0}^{\phi, k}$ and $E_{1}^{\phi, k}$ is less than a threshold, set $k=k-1$ and go to $(3)$

7. Define $\mathbf{F}^{\phi, k}$ as the oblique projector of nullspace $E_{0}^{\phi, k}$ and image $E_{1}^{\phi, k}$ (Eq. 2).

\subsubsection{Discussion}

This process is an intermediary between the original AFOP solution and the solution of conducting a PCA and removing the principal components. The AFOP solution ensures minimum removal of brain signal but with the risk of some instability. Whereas the PCA solution removes more signal and probably more brain signal but without the risk of instability due to the orthogonality and the fact that $\|F\|=1$. Any intermediary between those two solutions has the two constants:

- the number of removed components is the same;

- the two subspaces $E_{0}$ and $E_{1}$ are $\mathbf{C}^{\phi^{-1}}$-orthogonal which means that the rebuilt channels are the results from a least squares optimisation over the original signal.

The method automatically determines wether it should be closer to the AFOP solution or to the PCA solution. The PCA solution can be reached only when the artifacts are very high compared to signals of interest. In this case, the principal components are mainly composed of artifacts and the solution of removing them makes sense even if it would be suboptimal.

The mathematical demonstration of the statistical improvement of this orthogonalisation process remains a challenge. Consequently, a synthetic signal is presented to show the efficiency. 


\subsubsection{Synthetic signal}

Let us consider a known mixing matrix $\mathbf{M}$ (eq. 8) and two signal matrices with five sources $\mathbf{S}_{i m p}$ and $\mathbf{S}_{a r t}$ containing 10,000 temporal samples each. The first two sources represent artifacts and the last three represent brain sources. The brain sources $\mathbf{S}_{i m p}$ are generated with centred Gaussian law of standard deviation 1 and the artifact sources $\mathbf{S}_{\text {art }}$ are generated with centred Gaussian law of standard deviation [2 3]. The two raw signal matrices are then defined: the rest period $\left(\mathbf{V}_{i m p}=\mathbf{M} \mathbf{S}_{i m p}\right)$ and the artifact period $\left(\mathbf{V}_{\text {art }}=\mathbf{M} \mathbf{S}_{\text {art }}\right)$ using the mixing matrix:

$$
\mathbf{M}=\left(\begin{array}{ccccc}
1 & 0 & 1 & 0 & 3 \\
1 & 1 & -1 & 0 & 2 \\
1 & 0 & 0 & 1 & 1 \\
1 & 1 & 0 & 1 & 1 \\
1 & 0 & 0 & -1 & 0
\end{array}\right)
$$

With these matrices, the first step of AFOP gives the estimation of $E_{0}$ (the subspace generated by the first two columns of $\mathbf{M}$ ). Then, a second mixing matrix $\mathbf{M}^{\phi}$ is taken (eq. 9). The first two columns have the same mixing with small differences but the three others representing brain signals have a completely different mixing.

$$
\mathbf{M}^{\phi}=\left(\begin{array}{ccccc}
1.2 & 0 & 1 & 2 & -1 \\
0.9 & 1 & 2 & -1 & 0 \\
1 & 0.1 & 0 & 1 & 1 \\
1 & 1 & 0 & -1 & 1 \\
1 & 0.1 & -1 & 2 & 2
\end{array}\right)
$$


Another signal matrix of five sources $\mathbf{S}^{\phi}$ with 10,000 temporal samples is generated by Gaussian law with standard deviation 2. and 6. for artifact sources and 4., 1. and 1. for cerebral ones. The channel signal matrix is then $\mathbf{V}^{\phi}=\mathbf{M}^{\phi} \mathbf{S}^{\phi}$

The theoretical filtering matrix is given by $\mathbf{F}^{\phi}=\mathbf{M}^{\phi} \mathbf{D} \mathbf{M}^{\phi^{-1}}$ with $\mathbf{D}$ the diagonal matrix with values $\left[\begin{array}{lllll}0 & 0 & 1 & 1 & 1\end{array}\right]$ on the diagonal. The estimated filtering matrix $\mathbf{F}_{A F O P}^{\phi}$ corresponds to the matrix obtained with the standard AFOP method and the estimated filtering matrix $\mathbf{F}_{A F O P}^{\phi, 3}$ corresponds to the matrix obtained with the AFOP method and progressive orthogonalisation with $k=3$. Figure 3 illustrates the improvement of the stabilisation process on an extraction of these signals. It can be noticed that without stabilisation small perturbations have created an important error on the filtering signals, but this error is greatly reduced with the stabilisation process. To prove this improvement, the normalised mean squared differences is computed (eq. 10).

$$
Q(X, Y)=\frac{\sum_{i, j}\left(X_{i, j}-Y_{i, j}\right)^{2}}{\sum_{i, j} X_{i, j}^{2}}
$$

This difference is computed without stabilisation: $Q\left(\mathbf{F}^{\phi} \mathbf{V}^{\phi}, \mathbf{F}_{A F O P}^{\phi} \mathbf{V}_{t}\right)=$ 85\%, and with stabilisation: $Q\left(\mathbf{F}^{\phi} \mathbf{V}^{\phi}, \mathbf{F}_{A F O P}^{\phi, 3} \mathbf{V}_{t}\right)=15 \%$. The improvement is obvious.

\subsection{Overall process}

Each artifact does not appear on all frequency bands. Thus, for a specific frequency window, there is no need to use the filter of all types of artifacts. Consequently, the electrode artifact filter can be used only on windows $0-4 \mathrm{~Hz}$ and $4-8 \mathrm{~Hz}$, the ocular artifact filter can be used on windows $0-4 \mathrm{~Hz}, 4-8 \mathrm{~Hz}$ 
and $8-13 \mathrm{~Hz}$ and the muscle artifact filter can be used on windows $8-13 \mathrm{~Hz}, 13-$ $20 \mathrm{~Hz}$, and $20-45 \mathrm{~Hz}$. The stabilisation process can be used on all frequency windows but it is mostly useful for the window $0-4 \mathrm{~Hz}$.

For the frequency windows $0-4 \mathrm{~Hz}$ and $4-8 \mathrm{~Hz}$, both AFOP and DAFOP method have to be used to filter ocular and electrode artifacts. Applying the two different filtering matrices successively is not recommended since the product of two projection matrices is not a projection matrix. It is better to first remove ocular components via AFOP and then to apply DAFOP on only the remaining sources. Figure 2 illustrates all the details concerning filter combination.

\section{Clinical evaluation}

In this section, a first example illustrates the interest of each step (Fig. 4) of the global AFOP/DAFOP method. Subsequently, an evaluation of the performance of this method was carried out by quantifying the visual analysis performed by a neurologist. Finally, the results are compared with those obtained with manual ICA. Readers can also visualise a complete filtered

recording with the corresponding video on the author's website [http://www.samuelboudet.com/Vic

The dataset was supplied by the Hospital Group of the Catholic Institute of Lille (GHICL). Each recording was acquired with a 10/20 system with 19 electrodes from Nihon Khoden and Nicolet devices. A pre-process consisting of an average reference, a notch filter at $50 \mathrm{~Hz}$ for the sector, a high pass filter at $0.5 \mathrm{~Hz}$, and a low pass filter at $45 \mathrm{~Hz}$ was carried out before filtering. 


\subsection{Illustration of all steps}

Figure 4 represents an example where the filtering was particularly efficient. It illustrates the interest of the each iprovement of the method (Fig. 4B to E). This period contains alpha rhythm mainly on O1 and O2 as well as a spike-wave on second 3. The spike-wave was hidden by important ocular artifacts and it was very difficult even for a specialist to detect the spike-wave behind this artifact.

In this example, the standard AFOP method (Fig. 4-B) enabled the improvement of readability by removing most ocular artifacts while retaining the cerebral rhythms (alpha and spike-wave). It can be noticed however that a muscular artifact appeared on the frontal leads. The frequency window decomposition (Fig. 4-C) enabled the removal of this muscular artifact but there was a stability problem that prevented the removal of ocular artifacts. The orthogonalisation process (Fig. 4-D) removed this problem but there remained a tight slow artifact in the frontal electrodes. Eventually, the electrode artifact filter (Fig. 4-E) removed these last signs of slow artifacts. The brain signal appears to be entirely preserved. It can be noticed however that the spike (second 2.5) was possibly slightly reduced.

\subsection{Method evaluation}

In this study, a first evaluation was performed based on the evaluation of a neurologist's expertise which better corresponds to routine use. With no prior knowledge of the filtering results, the neurologist selected 330 artifacted pages of 20 s from 26 hours of recordings from 10 patients (see sec. 2.1). On each page, the neurologist noted what types of waves were present, as well as their importance (3 levels: small, mean, and important). Both artifact and brain 
signals were noted. Next, a visual inspection was performed on the filtered recording, and for each signal the neurologist quantified its elimination: $0=$ no reduction, $1=$ small reduction $(<30 \%), 2=$ mean reduction (between $30 \%$ and $70 \%$ ), $3=$ important reduction $(>70 \%), 4=$ complete elimination. The same notation was made for artifact and brain signals. Consequently, the ideal case would be 0 on brain signals and 4 for artifacts. The notation system is illustrated in Figures 5 and 6.

On the first example (Fig. 5(I)), spike-waves appeared between seconds 3 and 7. A mean muscular artifact can be observed in the frontal area as well as important ocular artifacts. This example is very representative of the mean result of the method: The spike-waves were only slightly reduced, the fast rhythms preceding the paroxysm (time: $3.8 \mathrm{~s}$ ) were perfectly visible, and the background rhythm was not modified. By contrast, the artifacts were highly reduced; there remained only a few ocular artifacts in the frontal area, as well as small muscular artifacts in frontal and temporal areas.

Figure 5(II) represents a period where the filtering was very efficient. An important muscular artifact in the temporal area was hiding pathological theta waves (seconds 0 to 4 in F7 and T3). After filtering, the muscular artifact was completely removed and the theta waves were emphasised. It can be noticed that there were also some small ocular artifacts (e.g. second 1) which were completely removed, and an important electrode artifact on O1 which was greatly reduced. The background rhythm, mainly composed of alpha, was unchanged.

Figure 5(III) shows a limitation of the method. A small delta slow wave appeared on F8 (second 2) and it was completely removed by the filter. 
Nevertheless, the alpha rhythm was still entirely preserved. There was also a small muscular artifact that was completely removed.

Figure 6(I) corresponds to an epileptic seizure. Several muscular, ocular and electrode artifacts were almost completely filtered out. The spikes were entirely preserved (electrodes F8, T4, T6). Delta waves appeared likewise between and were only slightly reduced.

Figure 6(II) represents the beginning of another epilepsy seizure period which was highly artifacted. The main parts of all artifacts were removed. Only the electrode artifact (second 5) remained due to its uncommonly high frequency. Regarding the cerebral signal, it was mainly composed of delta and theta rhythms which were entirely preserved by the filtering.

\begin{tabular}{|c|c|c|c|c|c|c|c|}
\hline $\begin{array}{l}\text { Small ocular } \\
\text { artifacts }\end{array}$ & $\begin{array}{l}\text { Important } \\
\text { ocular } \\
\text { artifacts }\end{array}$ & $\begin{array}{c}\text { Small } \\
\text { muscular } \\
\text { artifacts }\end{array}$ & $\begin{array}{l}\text { Mean } \\
\text { muscular } \\
\text { artifacts }\end{array}$ & $\begin{array}{l}\text { Important } \\
\text { muscular } \\
\text { artifacts }\end{array}$ & & $\begin{array}{c}\text { Small } \\
\text { electrode } \\
\text { artifacts }\end{array}$ & $\begin{array}{l}\text { Important } \\
\text { electrode } \\
\text { artifacts }\end{array}$ \\
\hline & $1 \% 8 \%$ & $1 \% \quad 1 \%$ & & & & $12 \%$ & \\
\hline $\begin{array}{lll}1 & 2 & 3 \\
(131 \text { pages) }\end{array}$ & $\begin{array}{ll}0 & \begin{array}{l}2 \\
\text { (120 pages) }\end{array}\end{array}$ & $\begin{array}{llll}0 & 1 & 2 & 3 \\
& (134 \text { pages) } \\
\end{array}$ & $\begin{array}{lll}0 & \begin{array}{l}1 \\
\text { (92 pages) }\end{array}\end{array}$ & \begin{tabular}{|ccc}
0 & 1 & 2 \\
& & 3 \\
& & 30 pages) \\
\end{tabular} & $\begin{array}{llll}0 & \begin{array}{l}1 \\
\text { (19 pages) }\end{array} \\
\end{array}$ & $\begin{array}{lll}0 & \begin{array}{l}1 \\
\text { (51 pages) }\end{array} \\
\end{array}$ & 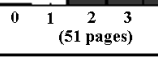 \\
\hline
\end{tabular}

Table 1: Amount of removed artifact per 20s page (0: no improvement, 1: small reduction $(<30 \%), 2$ : mean reduction (between $30 \%$ and $70 \%), 3$ : important reduction $(>70 \%), 4$ : complete elimination.

\begin{tabular}{|c|c|c|c|c|c|c|}
\hline Alpha & $\begin{array}{l}\text { Small } \\
\text { Delta }\end{array}$ & $\begin{array}{l}\text { Important } \\
\text { Delta }\end{array}$ & $\begin{array}{l}\text { Small } \\
\text { Theta }\end{array}$ & $\begin{array}{l}\text { Important } \\
\text { Theta }\end{array}$ & Spikes -waves & Spikes \\
\hline${ }^{770 \%} q_{11 \%}$ & $26 \% \%^{53 \%} 9 \%$ & $33 \% 56 \%$ & ${ }^{53 \%} 46 \%$ & $22^{20} 28 \%$ & $5 \% \quad 78 \%$ & $35 \%$ \\
\hline $0^{0} \underset{(203 \text { pages) }}{1}{ }^{12}{ }^{4}$ & 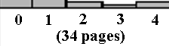 & \begin{tabular}{|c|c|}
0 & $\underset{(39 \text { pages) }}{2}{ }^{2}$ \\
4
\end{tabular} & 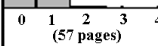 & 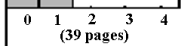 & 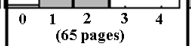 & 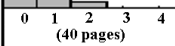 \\
\hline
\end{tabular}

Table 2: Amount of removed EEG per 20s page (0: no difference, 1: small reduction $(<30 \%), 2$ : mean reduction (between $30 \%$ and $70 \%), 3$ : important reduction $(>70 \%), 4$ : cannot be recognised

The overall results of this study are presented in Tables 1 and 2. As an 
example, it can be read as follows: among the 131 pages with small ocular artifacts, there was a complete elimination of this artifact in $71 \%$ of them, a small residue on $27 \%$ of them, ... Likewise, among the 203 pages with alpha rhythm, there is no attenuation of alpha on $87 \%$ of them, ...

\subsection{Comparison with ICA}

Today, ICA is probably the most widely accepted reference method for EEG artifact reduction. However, despite the large number of articles dealing with artifact reduction with ICA, we were unable to find any publication presenting a method which is simultaneously automated, reproducible and efficient for all artifacts. We thus decided to compare our results with manual ICA. It can be assumed that any method that aims at automating the ICA has at best the same results as the manual ICA method. Consequently, comparison with the AFOP/DAFOP method would be pertinent. Examining three methods, JADE [15], Infomax [16] and fastICA [17], we concluded that the method Infomax gave the best results. Consequently, a blind comparison between AFOP/DAFOP and Infomax was conducted by an expert neurologist.

52 artifacted pages among the previous 330 pages were selected for this comparison. This selection was done in order to best cover the variety of possible EEG rhythms and artifacts, and without viewing the results of each method. First, a neurologist (expert A) interpreted the rhythms and the artifacts of each raw page. Then, both AFOP/DAFOP and ICA filtering were applied. For the ICA filtering, the components were manually identified by two expert engineers (experts B and C) taking into account the neurologist's interpretation. The default parameters were those of the EEGLab[18] and 
20s windows were used for this filtering. Several tests were often conducted in order to obtain the best result for the component identification step. The number of remaining components varied from 4 to 16 with a mean of 11 , among the 18 possible. Then, for each page, the neurologist (expert A) scored the results of the two methods using the previously defined grid. The neurologist also noted which method was considered best for readability improvement. The results of the two methods were presented in random order in order to avoid expert subjectivity.

Table 3 sums up the results of this expertise. The two-sided sign test was applied to determine the significance of the difference between the methods for the elimination of each artifact type, for each cerebral rhythm, and in general. The Holm-Bonferroni correction $(\alpha=0.1)$ for hypothesis rejection was applied in order to take into account the fact that several tests were conducted.

Four examples were chosen to illustrates these results. The first example (Figure 4-F) shows the result of the manual ICA on the previously studied period. In this example the manual ICA preserved the cerebral waves, possibly slightly more than AFOP/DAFOP. However, the muscular artifact was not filtered out at all and there were still some ocular artifacts, contrary to the AFOP/DAFOP method.

Figure 7 represents an eye closing period of a patient with left frontotemporal epilepsy. An important alpha rhythm can be observed in channels $\mathrm{O} 1$ and O2, and pathological delta slow waves appeared in channels F7, T3, and T5. The pathological slow waves were partially hidden by a muscle artifact. In this case, the proposed method was very efficient and the entire EEG 


\begin{tabular}{|c|c|c|c|c|c|c|}
\hline Small ocular artifacts & 21 & 30 & 29 & 9 & 6 & 6 Unsignificant difference $(\mathrm{p}=0.61)$ \\
\hline Important ocular artifacts & 21 & 3,1 & 3,0 & 8 & 7 & 6 Unsignificant difference $(p=0.79)$ \\
\hline Small muscular artifacts & 20 & 3,6 & 2,7 & 15 & 4 & 1 AFOP is better than ICA $(p=0.00052)$ \\
\hline Mean muscular artifacts & 19 & 3,4 & 2,4 & 17 & 1 & 1 AFOP is better than ICA ( $p=0.00014)$ \\
\hline Important muscular artifacts & 10 & 3,2 & 2,4 & 8 & 2 & 0 AFOP is better than ICA ( $p=0.0078)$ \\
\hline Small electrode artifacts & 8 & 3,8 & 3,4 & 1 & 6 & 1 \\
\hline Important electrode artifacts & 9 & 3,8 & 3,8 & 2 & 5 & $\left.{ }_{2}\right\}$ Unsignificant difference $(p=1)$ \\
\hline Chewing artifacts & 3 & 2,3 & 2,7 & 1 & 1 & 1 Unsignificant difference $(p=1)$ \\
\hline All artifacts & 69 & 3,3 & 2,8 & 44 & 19 & 6 AFOP is better than ICA $\left(p=1.27 \mathrm{e}^{-6}\right)$ \\
\hline Alpha & 34 & 0,2 & 0,5 & 10 & 22 & 2 AFOP seems a bit better but there is no significance ( $p=0.038)$ \\
\hline Small Delta & 8 & 1,5 & 1,0 & 1 & 2 & 5 , \\
\hline Important Delta & 11 & 1,0 & 0,9 & 2 & 5 & eems a bit better but there is no significance $(p=0.146)$ \\
\hline Small Théta & 10 & 1,0 & 0,8 & 2 & 3 & 5 \} \\
\hline Important Théta & 12 & 1,0 & 0,8 & 2 & 6 & $\left.{ }_{4}\right\}$ Unsignificant difference $(p=0.267)$ \\
\hline Spikes & 12 & 0,8 & 0,6 & 4 & 2 & 6 \\
\hline Spike-waves & 10 & 0,4 & 0,6 & 2 & 8 & $\left.{ }_{0}\right\}$ Unsignificant difference $(p=1)$ \\
\hline $\begin{array}{l}\text { All pathological signals }(\boldsymbol{\Delta}, \boldsymbol{\theta}, \\
\text { spike,spike-waves) }\end{array}$ & 63 & 0,93 & 0,78 & 13 & 26 & 24 ICA seems a bit better but there is no significance $(p=0.099)$ \\
\hline All cerebral signals & 97 & 0,7 & 0,7 & 23 & 48 & 26 Unsignificant difference $(\mathrm{p}=0.775)$ \\
\hline Compilation of both cerebral & 208 & & & 67 & 67 & 32 AFOP is better than ICA $(p=0.000562)$ \\
\hline General for entire pages & 52 & & & 19 & 10 & 23 Unsignificant difference $(p=0.64)$ \\
\hline
\end{tabular}

Table 3: Results of the expert's blind comparison between AFOP/DAFOP process and ICA (infomax) filtering

signal was preserved while all the muscular artifacts were eliminated. The manual ICA methodwas unable to completely remove the muscular artifact and the alpha rhythm was greatly reduced. Nevertheless, the delta rhythm was entirely preserved.

Figure 8 represents a paroxysm period (second 4 -5) with a small ocular artifact (on FP1 and FP2 second 1) and an electrode artifact in second 6 . The proposed method filtered well the artifact but a reduction of the spike- 
wave can be observed in the frontal area. Here, the manual ICA gave better results since the brain signal was entirely preserved. This is due to the fact that only two components were removed by ICA whereas a fixed amount of components were removed by the AFOP/DAFOP method, even if there was no artifact.

\section{Discussion}

\subsection{Clinical evaluation}

After the examination of the 330 pages (Tables 1 and 2), the readability was judged better for $78 \%$ of pages, equal for $20 \%$ of pages and worse for $2 \%$. On 12 of the pages, an element was revealed that was not identifiable before filtering, particularly during seizures, whereas there were only 3 pages in which an element was completely removed and in which the interpretation was degraded.

According to the results presented in Table 1, on $95 \%$ of the pages the cerebral signals were preserved with more than $70 \%$ of their amplitude. In the same way, Table 2 shows that for $90 \%$ of pages, the artifacts were removed by more than $70 \%$ providing evidence of the efficiency of the method.

As can be observed in various samples, the alpha rhythm was always very well preserved (no modification can be seen in $87 \%$ of pages) (e.g. Fig. 4E, 5(III)-B, 7-B and 9-B). The theta rhythm was also well preserved even though a small reduction was often visible (the reduction was low or null in $98 \%$ of cases) (e.g. Fig. 5(II)-B, 6(II)-B). The delta rhythm was well

preserved most of the time (e.g. Fig. 6(I)-B, 6(II)-B, 7-B) despite a regular small extenuation. 
It can be noticed that the lower the frequency of an EEG rhythm, the more difficult its preservation, particularly over the frontal area. Six of the 73 examined pages with delta showed important reductions of this rhythm (e.g. Fig. 5(III)-B), which could skew the clinical analysis. Nevertheless, for the other $90 \%$, even if there was a reduction, the interpretation was unchanged. Most of the times, the spike-waves were slightly decreased, but in all cases, they remained entirely identifiable (e.g. Fig. 4-E, 5(I)-B, 8-B). The decrease was generally in the frontal area. The spikes were on average reduced by $30 \%$, whereas the waves were reduced by $20 \%$. On the two recordings containing isolated spikes, the spikes were always well preserved (e.g. Fig. 5(I)-B) (low or null reduction in $90 \%$ of cases, and mean reduction in the last 10\%).

Regarding the artifacts, they were all well filtered with the exception of the chewing artifact which was decreased only by $50 \%$. The chewing artifact is particularly fussy because it leads to a fast electrode artifact, very important on all channels and with an unstable location.

The ocular artifacts were filtered on average at $80 \%$ (e.g. Fig. 4-E, 5(I)-B, 6(I)-B, 6(II)-B, 8-B, 9-B). Nonetheless, some instability phenomena persisted when the ocular artifacts were important. Such instability might create a rhythm that could be wrongly interpreted as pathological. Most of time, the progressive orthogonalisation process reduced this phenomenon, but in some unusual rhythms remained in exceptional cases (3 pages among the 251 analysed pages with ocular artifacts).

The method was particularly efficient in filtering muscular artifacts (e.g. Fig. 7-B, 8-B, 8(I)-B, 8(II)-B, 9(I)-B, 9(II)-B) even when they were very important (reduction of $70 \%$ in $96 \%$ of cases). Concerning the electrode 
artifacts, they were completely filtered out in $70 \%$ of cases (e.g. Fig. 7-B, 8(II)-B, 9(II)-B). This filtering was however not effective when the artifact remained below a threshold, in which case there was no filtering at all. When the artifact came from an electrode or wire movement, it was strongly filtered most of time and when it came from a head movement, it propagated more and was just decreased in amplitude.

Further validation, particularly concerning epilepsy seizures, is of course necessary. With this aim, an expert committee has been created to perform a blind analysis with with a large volume of recordings. However, this first study can be considered as a representative sample of the results.

It would be possible to use the EEG filtering in contexts other than epilepsy. Some trials have been performed in dementia [19]. About 100 short-duration recordings have been reviewed by a neurologist and the results seem similar to those found in epilepsy. Some trials have also been carried out during sleep. It is possible to efficiently filter muscle and ocular artifacts, but unfortunately the electrode artifact filter can easily remove some slow rhythms during slow sleep. Further improvements will be required to have a suitable filter in this context.

\subsection{Comparison with ICA}

The results obtained with the present method were then compared with manual ICA in order to compare its performance with the best results achievable with ICA. Components were selected knowing exactly how the evaluator would interpret the various signals as artefact or brain signal. Consequently, it can be assumed that this identification step was very close to optimal and that any method which could blindly automate this step would produce, at 
best, the same results. Infomax was tested with specific parameters. Other good solutions may exist but Infomax was considered to be one of the best ICA methods and therefore tested alone.

From a general point of view, global comparison of each page did not show one method superior to the other. Analysis of the details of the rhythms showed that the AFOP/DAFOP method was significantly better for muscular artifact filtering. It also seemed to be better for the preservation of alpha rhythms but the significance is not validated by the Holm-Bonferroni method. The ICA seemed to be a bit better for the preservation of pathological slow rhythms, but the difference did not reach significance. The AFOP/DAFOP method seemed to be better because it took into account the compilation of both brain signals and artifacts, but any assessment of performance would depend on the priority of the application: artefact removal or preservation of pathological signals.

The better results observed with the AFOP/DAFOP method for muscular artifact filtering and alpha rhythm preservation were probably due to the frequency window decomposition. This decomposition could also be used to improve ICA results but the separation criteria might not necessarily be efficient on a limited frequency window. Moreover, the component identification step would be even more difficult.

For a truly objective comparison, the filtering should be carried out by the authors of the ICA methods with several evaluating experts and a larger number of pages. These early trials are nevertheless sufficient to demonstrate the competitive performance level of the AFOP/DAFOP method. 


\subsection{Practical aspects}

A first advantage of this method is its automation, and the possibility of filtering hours of recording without manual intervention. Nevertheless, a learning period is still required to learn the artifact distribution. Our first trials show that it would be possible to consider that the artifact distribution is constant enough for all subjects, but for now it is recommended to perform a learning period at the beginning of each recordings.

A second advantage is the very short processing time $(<0.2 s$ for a $25 \mathrm{~s}$ page on Matlab using a dual core $2 \mathrm{GHz}$ processor). Thus, this filter can be used for real time exploitation and can also be applied when the recordings are visualised (in the routine practice, a neurologist analyses about three $20 \mathrm{~s}$ pages per second). Parameters can be then individuality adapted for pages of interest with no lag time.

Another advantage is that the method does not require the addition of the ocular electrodes not widely used for clinical recordings.

At the present time, one limitation could be the constant number of removed components over the entire recording, meaning the method filters signals even if there is no artifact. Consequently, there is a small but real reduction of brain signals on pages with no artifacts. This might be avoided.

Regarding the programs, the authors will try to promote the method with the EEG device producer. Thus, for neurologists, this method could be available in the near future for use directly on the monitor. For researchers, a free version of the Matlab programs will be discussed with the producer. For now, future users should contact the authors to obtain the programs. 


\section{Conclusion}

This paper presents a robust method to filter most of types of artifacts found in EEG recordings. To sum up, the AFOP/DAFOP method consists of comparing two distinct time-frequency windows and performing a regression on a third with potentially a dimension reduction by PCA in order to stabilise the process. It is interesting to notice that the same process can be used to filter a very large variety of artifacts, and many applications can be imagined (e.g. [14]). There are three possible uses of this method to filter artifacts by function of their types:

- the first one [10] consists of working within a given frequency window, comparing two time windows for the first step, and performing a regression on a third time window for the second step. The method must be used when the spatial projection of either signals of interest or artifact is constant. In this paper this method is used to filter ocular and muscular artifacts;

- the second one is the dual frequency method of the first. It consists of working within a given time window and comparing two frequency windows for the first step and filtering a third frequency window for the second step. It can be used if there is a prior knowledge about the frequencies of signals and when there is an important difference of variance ratio between two frequency bands. In this paper, this method is used to filter electrode artifacts, but it can also be used to filter muscular artifacts. Some trials have been performed, and results are encouraging; 
- the third one described in [1][2], consists of filtering periodic signal synchronised with an other like ECG artifact.

A study has been performed using real cases of long duration recordings which often cause difficult interpretation problems due to the presence of artifacts. Compared with ICA-based methods, the results were promising. One of the best improvements was the use of time-frequency windows which can also be performed with ICA.

Various investigating neurologists found this filtering method noteworthy, on the one hand because of its efficiency for most artifact types, and on the other hand because of its sensitivity to both normal and pathological cerebral rhythms. It was specifically noted that it does not significantly distort paroxysmal activities: spikes, spike-waves...

The main drawback concerns preservation of the delta band where a marked decrease in amplitude can sometimes be observed, apparently more so than with infomax filtering. Conversely, artifact residue can occasionally lead to the production of slow rhythms in the frontal area which might be incorrectly interpreted as pathological if a neurologist analyses only the filtered EEG. These problems are however relatively uncommon.

The contribution of this method is obvious in the field of epileptology where filtering uncovers paroxysmal activities, particularly at the beginning of a seizure. Three points facilitating the clinician's diagnostic process can be emphasised: the method is automatic, the processing time is short and no additional electrodes are required. Such details have importance because they often represent limitations for the clinical use of EEG filters. 
We continue to improve the method, particularly concerning perfect preservation of the delta band rhythm and removal of non-existing rhythms on the frontal electrodes when filtering eyes. An interesting improvement could be to use filtering only when necessary, which would require combining the method with artifact detection (e.g. [20]). This would reduce the probability of removing EEG rhythms. We are also working on the deletion of the learning period that can be a bit restrictive if the protocol is not carried out at the beginning of a recording.

In other contexts, this method might also be used as a pre-process for Fourier transformation or non-linear analysis and might improve certain applications such as anticipated detection of epilepsy seizures, brain-computer interfaces, neurofeedback, or sleep analysis. Another prospect would be the application of this method to ambulatory recordings where the expected increase in artifacts creates a challenging filtering problem.

A patent [13] is pending for this method, and an expert committee has been created to carry out a complete evaluation of the method for possible clinical use.

\section{Acknowledgement}

The authors gratefully acknowledge the medical staffs of St Vincent and St Philibert Hospitals of Lille. They also acknowledge the financial support of FRM. 


\section{References}

[1] Y. Wang, Reduction of cardiac artifacts in magnetoencephalogram, Proceedings of the 12th International Conference on Biomagnetism (Biomag 2000) (2000).

[2] S. Boudet, L. Peyrodie, P. Galois, C. Vasseur, A robust method to filter various types of artifacts on long duration EEG recordings, in: Proc. of 2nd International Conference on Bioinformatics and Biomedical Engineering (IEEE-ICBBE), pp. 2357-2360.

[3] T. Lagerlund, F. Sharbrough, N. Busacker, Spatial filtering of multichannel electroencephalographic recordings through Principal Component Analysis by Singular Value Decomposition, Journal of Clinical Neurophysiology 14 (1997) 73-82.

[4] R. Croft, R. Barry, Removal of ocular artifact from the EEG : a review, Clinical Neurophysiology 30 (2000) 5-19.

[5] T. Jung, S. Makeig, C. Humphries, T. Lee, M. M.J., V. Iragui, T. Sejnowski, Removing electroencephalographic artifacts by blind source separation, Psychophysiology 37 (2000) 163-178.

[6] T. Liu, D. Yao, Removal of the ocular artifacts from EEG data using a cascaded spatio-temporal processing, Computer methods and programs in biomedicine 83 (2006) 95-103.

[7] F. Campos Violaa, J. Thornea, B. Edmondsc, T. Schneiderd, E. T., S. Debenera, Semi-automatic identification of independent components representing eeg artifact, Clinical Neurophysiology 120 (2009) 868-877. 
[8] C. James, O. Gibson, Temporally constrained ICA: an application to artifact rejection in electromagnetic brain signal analysis, IEEE Trans. on Biomedical Engineering 50 (2003) 1108-1116.

[9] P. Le Van, E. Urrestarazu, J. Gotman, A system for automatic artifact removal in ictal scalp EEG based on independent component analysis and Bayesian classification, Clinical Neurophysioly 117 (2006) 912-927.

[10] S. Boudet, L. Peyrodie, P. Gallois, C. Vasseur, Adaptive Filtering by Optimal Projection and application to automatic artifact removal from EEG, Signal Processing 87 (2007) 1978-1992.

[11] P. Common, Independent component analysis, a new concept, Sig. Pro. 36 (1994) 287-314.

[12] K. K. Ang, Z. Y. Chin, H. Zhang, C. Guan, Filter bank common spatial pattern (fbcsp) in brain-computer interface, in: IEEE International Joint Conference on Neural Networks(IJCNN)., pp. 2390 -2397.

[13] S. Boudet, L. Peyrodie, P. Gallois, Patent : PCT/FR2009/051340, Procédé de traitement de données permettant le filtrage d'artefacts, adapté notamment à l'électroencéphalographie, Technical Report, 2009.

[14] R. Sameni, C. Jutten, M. Shamsollahi, A Deflation Procedure for Subspace Decomposition, IEEE Trans. on signal processing 58 (2010) 23632374 .

[15] J. Cardoso, High-order contrasts for independent component analysis, Neural Computation 11 (1999) 157-192. 
[16] T. Bell, T. Sejnowski, An information-maximization approach to blind separation and blind deconvolution., Neural Comutation 7 (1995) 10041034 .

[17] A. Hyvrinen, E. Oja, A fast fixed-point algorithm for independent component analysis, Neural Computation 9 (1997) 1483-1492.

[18] A. Delorme, S. Makeig, EEGLAB: an open source toolbox for analysis of single-trial EEG dynamics including independent component analysis, J. Neuroscience Methods 134 (2004) 9-21.

[19] J. Gallois, Analyse linéaire et non linéaire de l'EEG dans les démences, Ph.D. thesis, Université de Lille 2, 2008.

[20] A. Delorme, T. Jung, T. Sejnowski, S. Makeig, Improved rejection of artifacts from EEG data using high-order statistics and independent component analysis, Neuroimage 34 (2007) 1443-1449. 


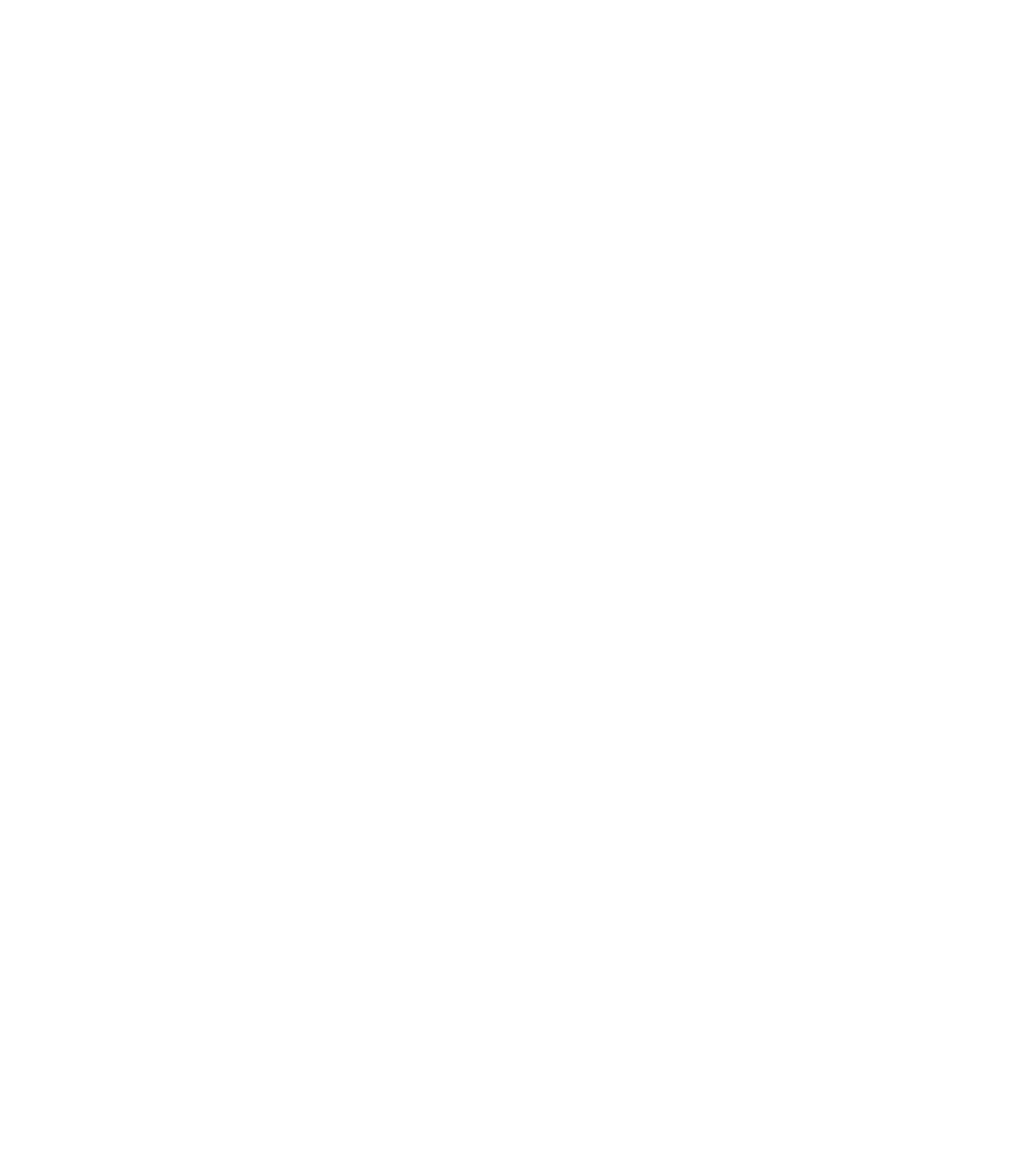

Figure 2: Overall method for filtering all types of artifacts 


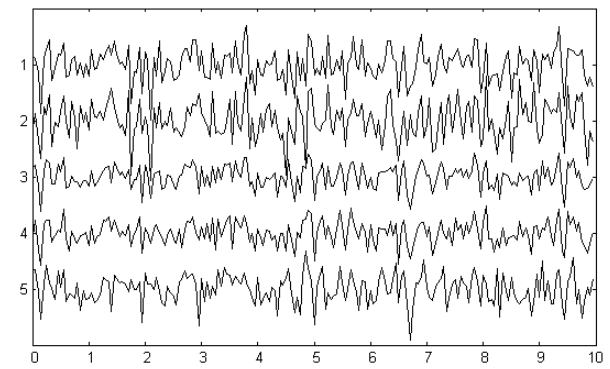

(a) Original artifacted signals

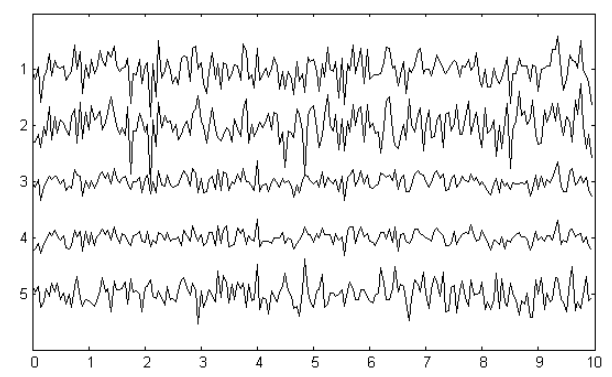

(c) Application of standard AFOP pro$\operatorname{cess} \mathbf{F}_{A F O P}^{\phi} \mathbf{V}^{\phi}$

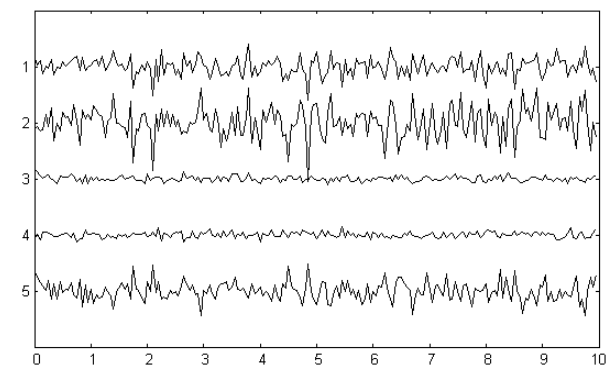

(b) Application of theoretical filtering $\operatorname{matrix} \mathbf{F}^{\phi} \mathbf{V}^{\phi}$

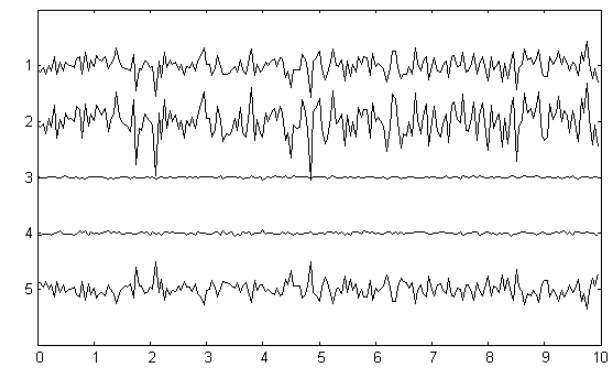

(d) Application of AFOP and progressive orthogonalisation process $\mathbf{F}_{A F O P}^{\phi, 3} \mathbf{V}^{\phi}$

Figure 3: Comparison of filtering result for synthetical samples 



Figure 4: Example of filtering with all methods: spike-wave and important ocular artifact:

(A) original signals, (B) filtering with AFOP, (C) filtering with AFOP on frequency band decomposition, (D) filtering with stabilised AFOP on frequency band decomposition, (E) filtering by the complete method, (F) filtering by manual ICA (Infomax - 8 remaining components) 
$\mathbf{A}$

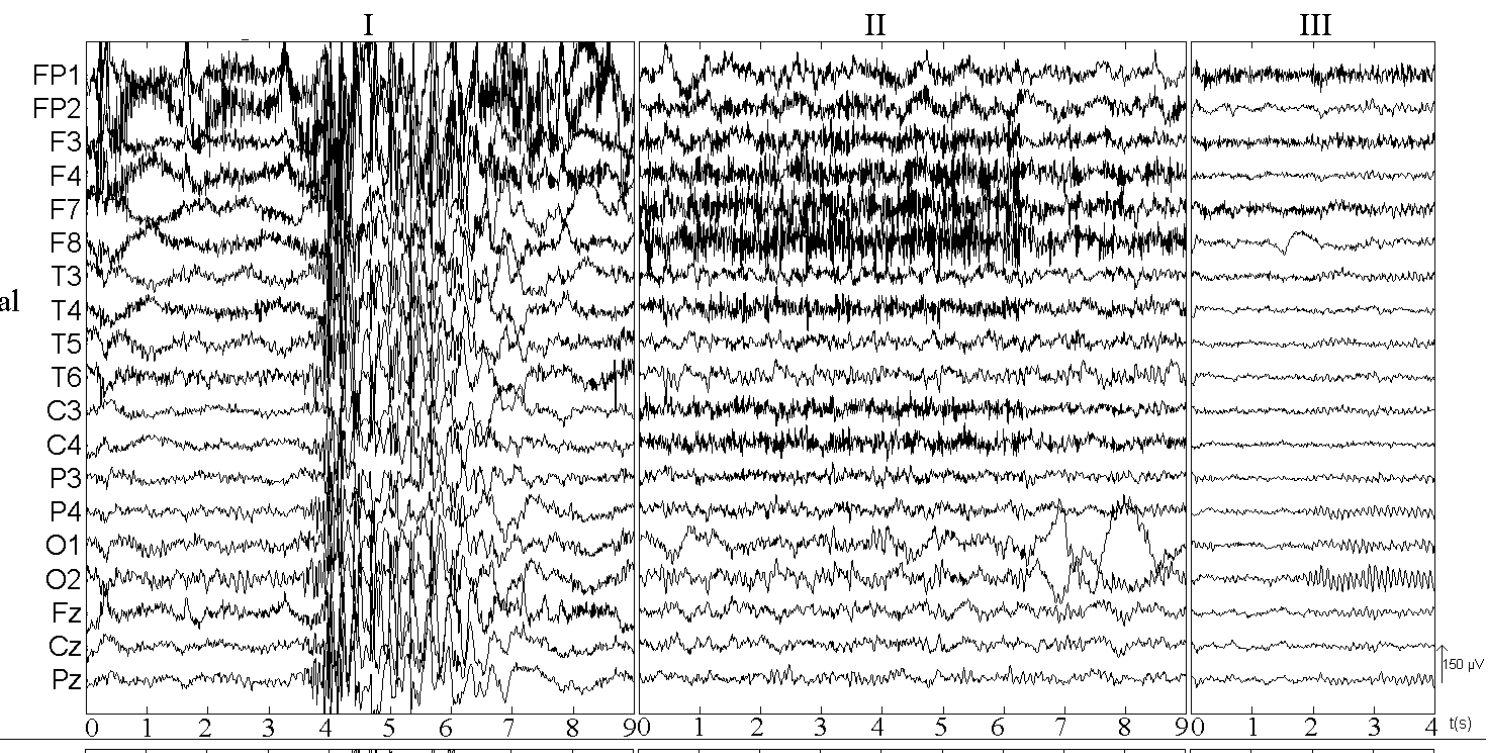

$\overline{\mathbf{B}}$

Original

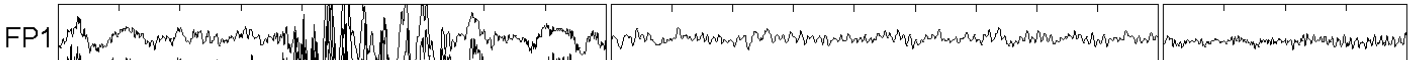

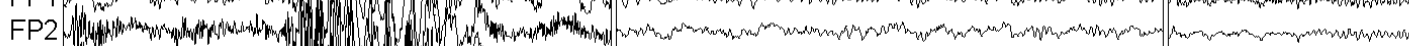

F3.

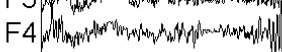

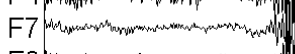

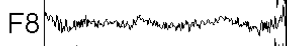

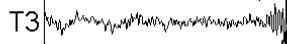

Filtered

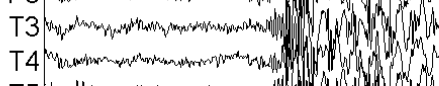

EEG

T5 5 in

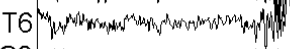

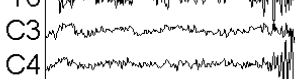

C4 4 rim

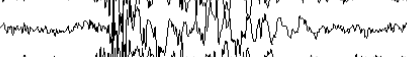

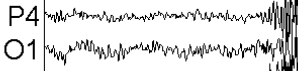

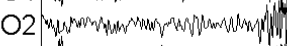

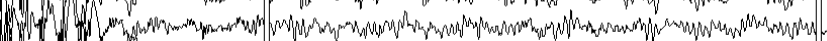

Fz

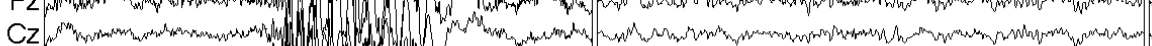

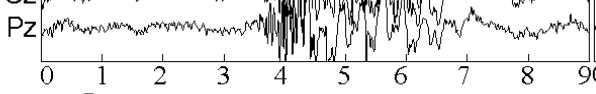

I

Neurologist

\begin{tabular}{|c|l|}
\hline Signal type & Elimination \\
\hline
\end{tabular}

evaluation:

Mean muscular artifacts $3(>70 \%)$

Important ocular artifacts $3(>70 \%)$

Spike-waves

$1(<30 \%)$

II

\begin{tabular}{|l|l|}
\hline \multicolumn{1}{|c|}{ Signal type } & Elimination \\
\hline Important muscular artifacts & $3(>70 \%)$ \\
\hline Small ocular artifacts & $3(>70 \%)$ \\
\hline Important electrode artifacts & $4(\approx 100 \%)$ \\
\hline Alpha rhythm & $0(\approx 0 \%)$ \\
\hline Small theta rhythm & $0(\approx 0 \%)$ \\
\hline
\end{tabular}

III
\begin{tabular}{|l|l|}
\hline \multicolumn{1}{|c|}{ Signal type } & \multicolumn{1}{c|}{ Elimination } \\
\hline Small muscular artifacts & $4(\approx 100 \%)$ \\
\hline Alpha rhythm & $0(\approx 0 \%)$ \\
\hline Small delta waves & $4(\approx 100 \%)$ \\
\hline
\end{tabular}

Figure 5: Filtering examples with signal elimination quantification by neurologist between

(A) original EEG and (B) filtered EEG. 


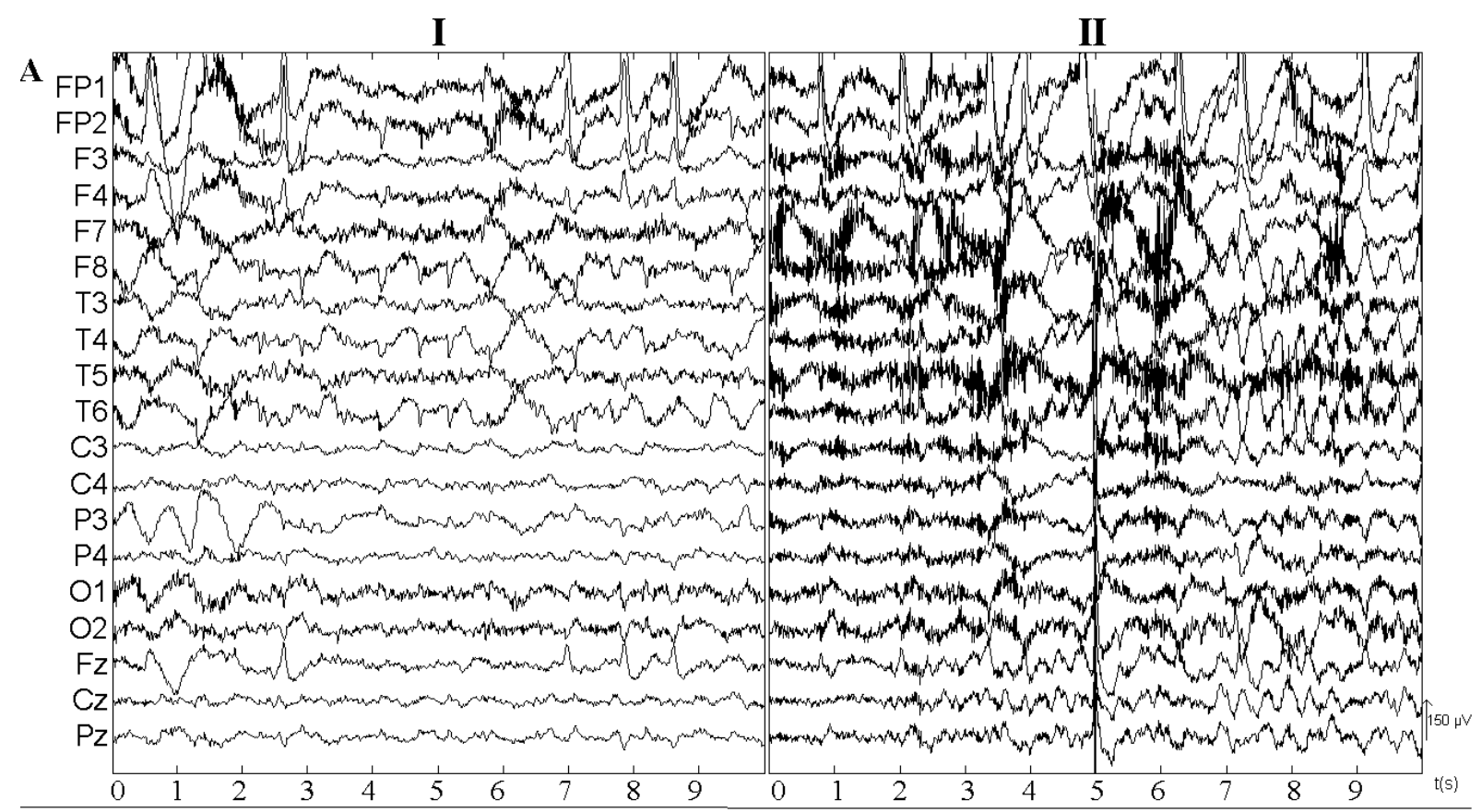

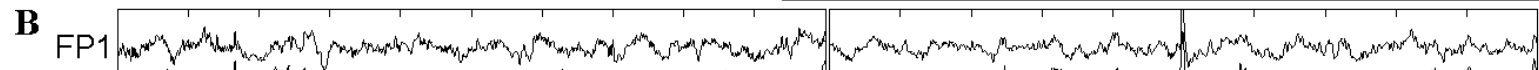
FP2 2.

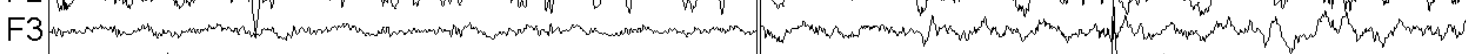

F4 W

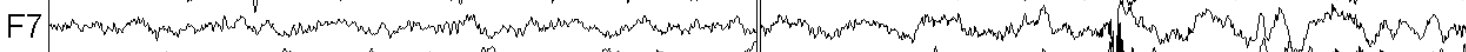

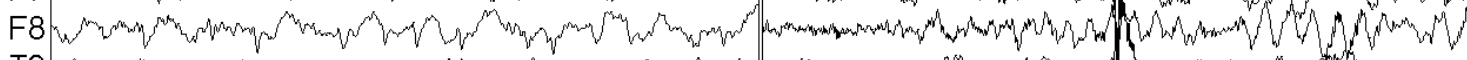

T3 14.

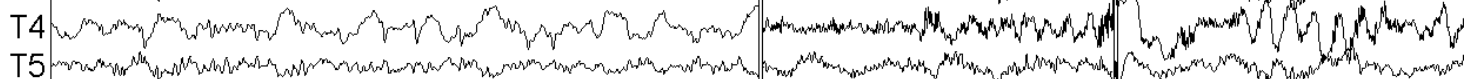

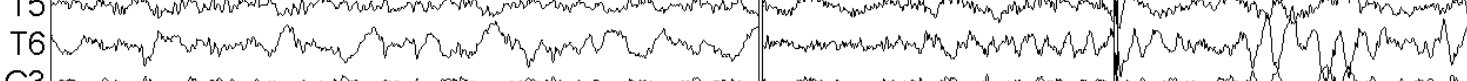

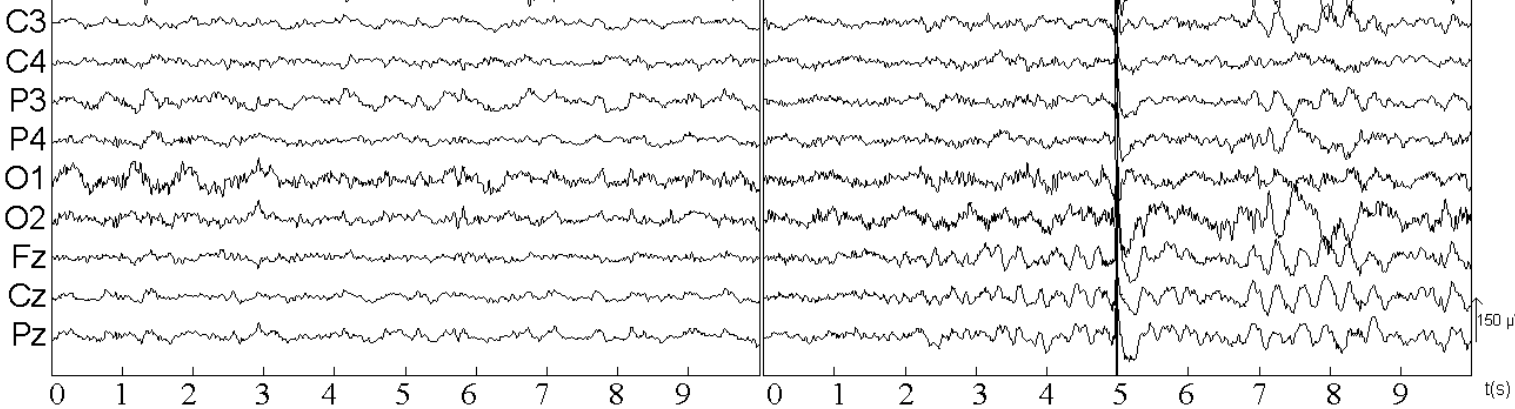

\begin{tabular}{|c|c|c|c|c|}
\hline \multirow{7}{*}{$\begin{array}{l}\text { Neurologist } \\
\text { evaluation: }\end{array}$} & & & & \\
\hline & Signal type & Elimination & \begin{tabular}{|c} 
Signal type \\
\end{tabular} & Elimination \\
\hline & Important ocular artifacts & $3(>70 \%)$ & Important ocular artifacts & $3(>70 \%)$ \\
\hline & Mean muscular artifacts & $3(>70 \%)$ & Important muscular artifacts & $3(>70 \%)$ \\
\hline & Small electrode artifacts & $4(30-70 \%)$ & Important electrode artifacts & $2(30-70 \%)$ \\
\hline & Important delta rhythm & $1(\approx 0 \%)$ & Important delta rhythm & $0(\approx 0 \%)$ \\
\hline & Spikes & $0(\approx 0 \%)$ & Small theta & $0(\approx 0 \%)$ \\
\hline
\end{tabular}

43

Figure 6: Filtering examples of epilepsy seizures with signal elimination quantification by neurologist between (A) original EEG and (B) filtered EEG. 

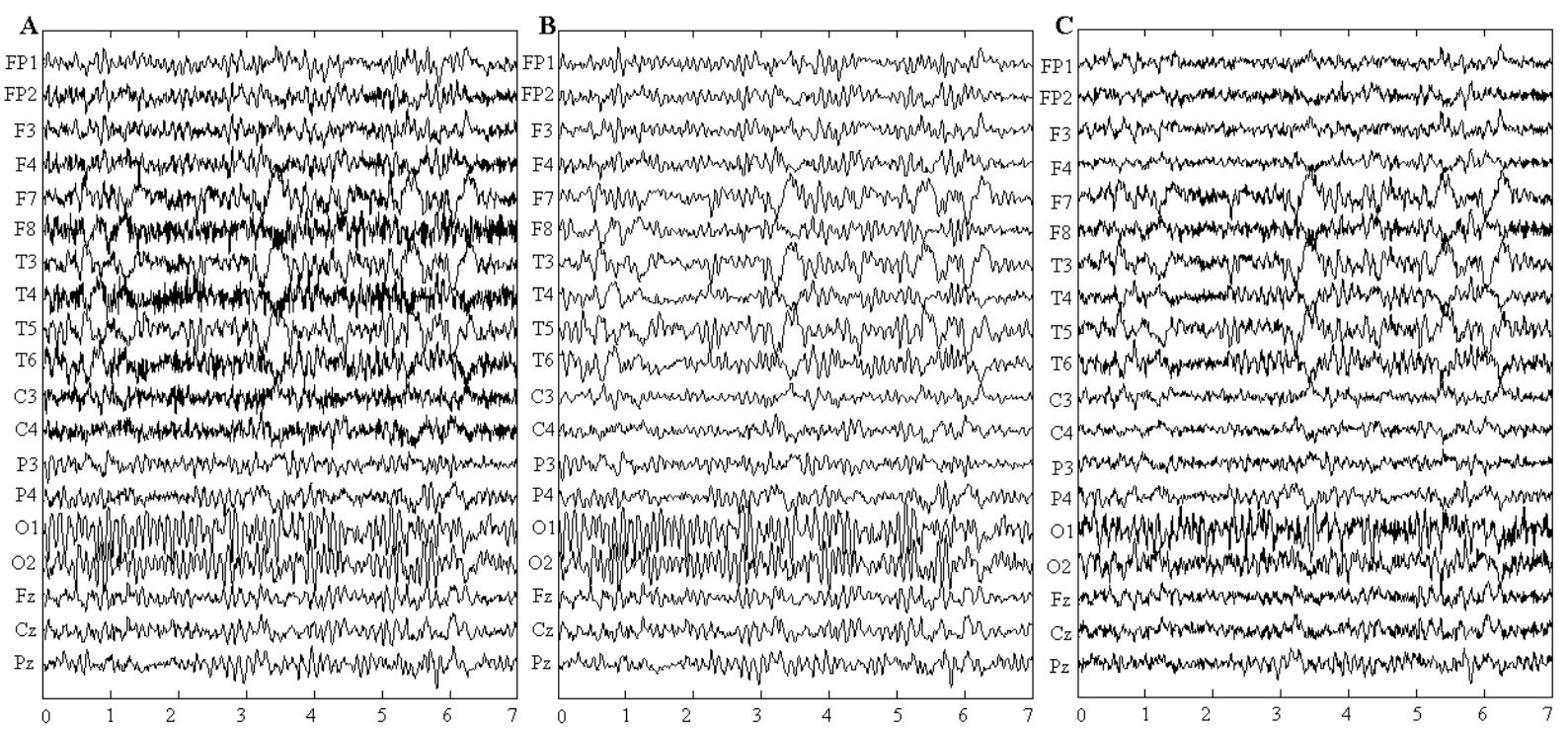

Figure 7: Example of filtering: alpha rhythm and delta pathological slow waves covered by a muscle artifact; (A) original signals, (B) filtering by the complete method, (C) filtering by manual ICA (infomax- 13 remaining components.)
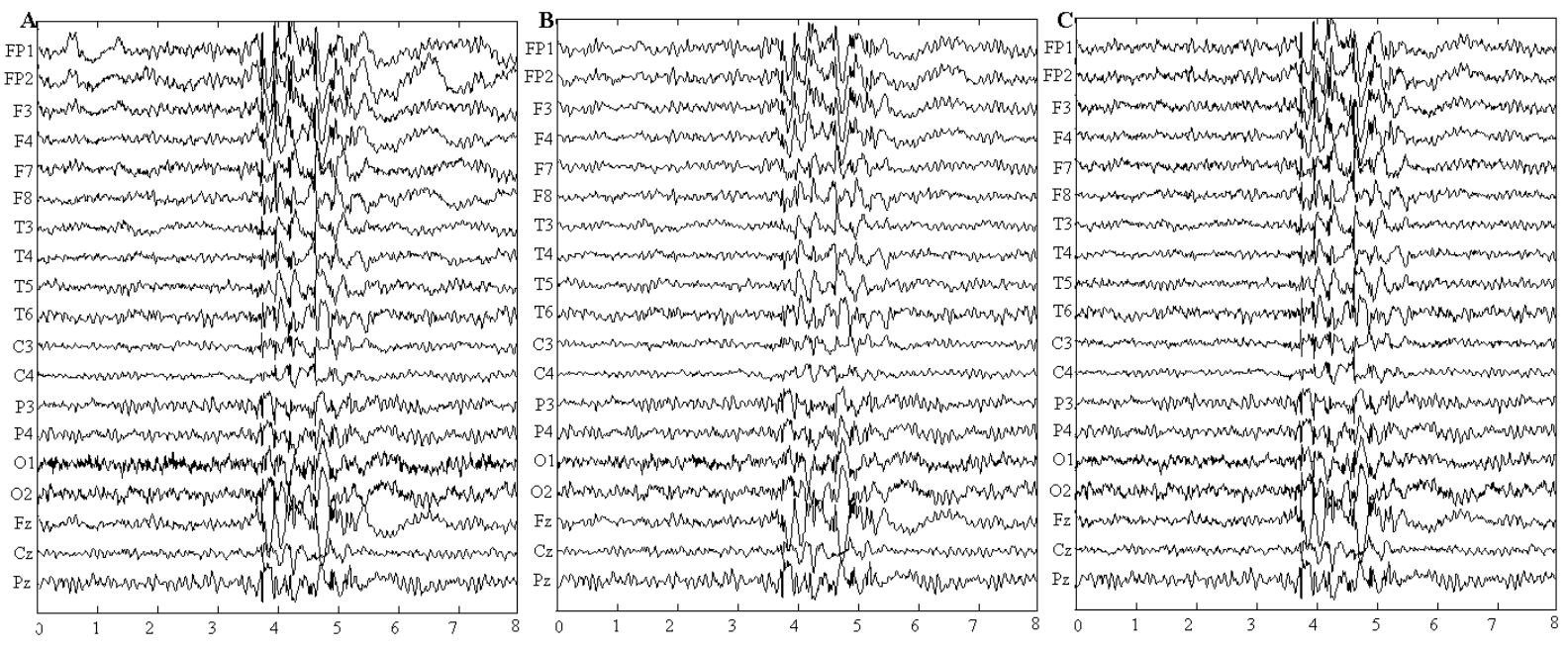

Figure 8: Example of filtering: Spike-waves and small ocular artifact; (A) original signals (B) filtering by the complete method (C) filtering by manual ICA (infomax- 16 remaining components.) 